\title{
GSK1838705A, an IGF-1R inhibitor, inhibits glioma cell proliferation and suppresses tumor growth in vivo
}

\author{
XIANG ZHOU ${ }^{1}$, FAZHENG SHEN ${ }^{1}$, PENGJU MA ${ }^{1}$, HONGYAN HUI ${ }^{2}$, SUJUAN PEI ${ }^{2}$, \\ MING CHEN $^{2}$, ZHONGWEI WANG ${ }^{1}$, WENKE ZHOU ${ }^{1}$ and BAOZHE JIN ${ }^{1}$ \\ Departments of ${ }^{1}$ Neurosurgery and ${ }^{2}$ Pharmacy, The First Affiliated Hospital of Xinxiang Medical University, \\ Xinxiang, Henan 453100, P.R. China
}

Received August 20, 2014; Accepted May 8, 2015

DOI: $10.3892 / \mathrm{mmr} .2015 .4129$

\begin{abstract}
Glioma is a type of primary malignant tumor of the central nervous system in humans. At present, standard treatment involves surgical resection, followed by radiation therapy and chemotherapy. However, the prognosis is poor and the long-term survival rate remains low. An improved understanding of the molecular basis for glioma tumorigenesis is in urgently required. The pro-survival effect of the insulin-like growth factor (IGF) signaling pathway has been implicated in progression of the glioma disease state. GSK1838705A is a novel, small molecule kinase inhibitor of IGF-IR, which inhibits IGF signal transduction and downstream target activation. Its anti-proliferative activity has been demonstrated in various tumor cell lines. The present study investigated the potential use of GSK1838705A for the treatment of glioma. Human U87MG glioma cells were used to examine the inhibitory activity of GSK1838705A in cell proliferation, migration and apoptosis. The antitumor activity of GSK1838705A was assessed in a xenograft mouse model. GSK1838705A inhibited the growth and induced the apoptosis of the U87MG glioma cells in a dose-dependent manner. The GSK1838705A-treated cells exhibited reduced migratory activity in response to chemoattractants. The present study further demonstrated the antitumor activity of GSK1838705A in vivo. The administration of GSK1838705A significantly inhibited the growth of glioma tumors by inducing the apoptosis of tumor cells. These results suggested that targeting IGF signaling with GSK1838705A may be a promising therapeutic strategy for the treatment of patients with glioma.
\end{abstract}

Correspondence to: Dr Baozhe Jin, Department of Neurosurgery, The First Affiliated Hospital of Xinxiang Medical University, 88 Jiankang Road, Weihui, Xinxiang, Henan 453100, P.R. China E-mail: baozhejin@gmail.com

Key words: glioma, GSK1838705A, insulin-like growth factor, signal transduction, small molecule kinase inhibitor, targeted cancer therapy

\section{Introduction}

Glioma is a collective group of brain tumors, which can occur in adults and children. According to pathological evaluation of the tumor, glioma are further classified to low-grade and high-grade glioma. Low-grade glioma progresses slowly, and the patients have a median survival rate of 11-17 years $(1,2)$. However, patients with high-grade glioma, including anaplastic astrocytomas and glioblastoma multiforme, have a poor prognosis and a substantially lower 3-year relative survival rate despite the advances in understanding of tumor pathology and the development of cancer therapy $(3,4)$. At present, the standard treatment for glioma includes surgical resection, followed by radiation therapy and chemotherapy. Temozolomide, an alkylating drug capable of methylating DNA and causing DNA damage, is the most commonly used chemotherapeutic agent in glioblastoma multiforme and relapsed anaplastic astrocytomas $(5,6)$. However, chemoresistance occurs frequently and recurrence is common, therefore the 5-year survival rate remains low in these patients (3). Novel and effective chemotherapeutic and targeted therapeutic strategies are urgently required and are an area of continued investigation.

Dysregulated gene expression and aberrant signal transduction have been attributed to the pathogenesis and tumorigenesis of various types of human malignancies. Insulin-like growth factor (IGF) and its receptor IGF-IR are important in growth and development. Circulating IGF-1 and IGF-2 bind to the cell surface receptor IGF-IR and activate the downstream phosphoinositide 3-kinase (PI3K)/AKT and mitogen-activated protein kinase (MAPK) signaling pathways, both of which have well defined proliferative, anti-apoptotic and oncogenic transformative roles $(7,8)$. Increased levels of circulating IGF-1 and IGF-2 have been detected in patients with colorectal cancer, prostate cancer, breast cancer and ovarian cancer (9-12). In glioma, deregulated IGF signaling has been associated with progression of the disease state (13). The inhibition of IGF signaling by small interfering RNA, specific kinase inhibitors or antibodies, suppresses tumor cell growth and survival (14-16). Furthermore, IGF signaling is involved in cellular migration and invasion, with the observation that the tumor cells at the margin of infiltration exhibit higher expression levels of IGF-IR compared with low-grade tumor cells $(13,17)$. IGF signaling serves as an effective therapeutic 
target for glioma, as a result of the central role IGF/IGF-IR has in tumor survival, migration and metastasis.

GSK1838705A, also termed 1H-pyrrolo[2,3-d]pyrimidine, is a novel, small molecule kinase inhibitor of IGF-IR and anaplastic lymphoma kinase (ALK) $(18,19)$. GSK1838705A has been demonstrated to inhibit the proliferation of cells derived from a variety of types of human tumor, including multiple myeloma, neuroblastoma and certain subtypes of non-small cell lung cancer (18). The present study aimed to investigate the inhibitory effect of GSK1838705A on glioma tumor cell proliferation and migration, and the growth of tumor xenografts in vivo. The results may support the use of GSK1838705A as a potential antitumor drug for the treatment of human glioma.

\section{Materials and methods}

Reagents, cells and mouse model. GSK1838705A was purchased from Selleck Chemicals LLC (Houston, TX, USA). The human U87MG glioma cell line was purchased from American Type Culture Collection (Rockville, MD, USA). The U87MG cells were maintained in Eagle's minimal essential medium (EMEM), supplemented with $10 \%$ heat-inactivated fetal bovine serum (FBS) and $100 \mathrm{U} / \mathrm{ml}$ penicillin-streptomycin in a humidified incubator at $37^{\circ} \mathrm{C}$ with $5 \% \mathrm{CO}_{2}$. Athymic nude mice were purchased from Slack Company (Shanghai, China) and were handled in compliance with the Experimental Animal Care and Use Committee guidelines of Xinxiang Medical University (Xinxiang, Henan, China). A total of 18 female mice (age, 7-8 weeks; weight, 24-28 g) were housed in a specific-pathogen-free facility maintained between 20 and $25^{\circ} \mathrm{C}$ with a $12 \mathrm{~h}$ light/12 h dark cycle, and were allowed ad libitum access to food and water. The study was approved by the ethics committee of Xinxiang Medical University (Xinxiang, China).

Cell viability assay. The cells $\left(1 \times 10^{5}\right)$ were seeded into 96 -well plates in triplicate and were treated with dimethyl sulfoxide (DMSO) or different concentrations of GSK1838705A for 24,48 or $72 \mathrm{~h}$. Cell viability at the end of each treatment was measured using a CellTiter-Glo Assay kit (Promega Corporation, Madison, WI, USA), according to the manufacturer's instructions.

Flow cytometric analysis. The cells $\left(1 \times 10^{6}\right)$ were treated with DMSO or different concentrations of GSK1838705A. Following treatment for $48 \mathrm{~h}$, the cells were harvested, fixed and stained with propidium iodide using a CycleTEST plus DNA reagent kit (Becton Dickinson, Franklin lakes, NJ, USA), according to the manufacturer's instructions. Following staining, the cells were collected and processed using a FACSCalibur (BD Biosciences, San Jose, CA, USA). The DNA content was analyzed using CellQuest Pro flow cytometry analytical software (version 5.1; Becton Dickinson).

Nuclear staining. Exponentially growing cells $\left(5 \times 10^{5} / \mathrm{ml}\right)$ were seeded onto polylysine coated glass coverslips overnight and treated with DMSO or different concentrations of GSK1838705A for $48 \mathrm{~h}$. The cells were subsequently fixed with $4 \%$ paraformaldehyde for $10 \mathrm{~min}$ and stained with
Hoechst (Sigma-Aldrich, St Louis, MO, USA) for a further $10 \mathrm{~min}$. Images were captured using a fluorescent microscope (BX51; Olympus America Inc., Melville, NY, USA).

Transwell assay. A total of $1 \times 10^{5}$ cells in EMEM, supplemented with $1 \%$ FBS, were seeded into the upper compartment of a 24-transwell Boyden chamber (Costar, Bedford, MA, USA). Subsequently, DMSO or different concentrations of GSK1838705A were added to the cells. EMEM $(650 \mu 1)$, supplemented with $10 \%$ FBS, was loaded into the lower chamber for use as a chemoattractant. Following incubation for $12 \mathrm{~h}$ at $37^{\circ} \mathrm{C}$, the cells were fixed with $70 \%$ ethanol and stained with $0.1 \%$ crystal violet (Sigma-Aldrich). The non-migrating cells, which remained on the upper surface were removed by gentle scraping with a cotton swab and images of the migrated cells on the lower side were captured using an Olympus BH2 microscope (Olympus, Tokyo, Japan), following which the cells were lysed with $10 \%$ acetic acid. The absorbance was measured at $595 \mathrm{~nm}$ using a Beckman DU-640 spectrophotometer (Beckman Coulter, Pasadena, CA, USA).

In vivo efficacy investigations using a mouse xenograft model. Exponentially growing U87MG cells were injected into the axillary region of nude mice $\left(2 \times 10^{6}\right.$ cells/mouse). When the tumors reached $\sim 70 \mathrm{~mm}^{3}$ in size, the mice were randomly allocated into a control and a treatment group, each containing six animals. The mice in the control group were administered orally with formulating vehicle, consisting of $20 \%$ sulfobutyl ether $\beta$-cyclodextrin (ISP; pH 3.5; Sigma-Aldrich) and the mice in the treatment group were administered orally with 4 or $8 \mathrm{mg} / \mathrm{kg}$ GSK $1838705 \mathrm{~A}$ once daily. The mice were weighed and the tumor size was measured every other day for 11 days. The tumor volume was calculated using the following formula: Tumor volume $\left(\mathrm{mm}^{3}\right)=$ (width $\mathrm{x}$ width $\mathrm{x}$ length) $/ 2$. At the end of the treatment period, the mice were sacrificed via carbon dioxide inhalation by trained personnel, and the tumors were harvested for analysis.

Immunohistochemical analysis of tumor samples. The tumor samples were cut into $5 \mathrm{~mm}^{3}$ tissue sections and were immediately fixed in $10 \%$ neutral buffered formalin (Sigma-Aldrich), followed by transfer into $70 \%$ ethanol. Following fixation, the samples were embedded into paraffin blocks and sectioned at a thickness of $5 \mathrm{~mm}$. A terminal deoxynucleotidyl transferase dUTP nick end labeling (TUNEL) assay was performed using a FragEL kit (Calbiochem, Darmstadt, Germany), according to manufacturer's instructions. The nuclei were stained with Hoechst for $20 \mathrm{~min}$ and images were captured using an Olympus fluorescent microscope.

Statistical analysis. Student's t-test and analysis of variance were performed using StatView software version 5.0 (SAS Institute, Cary, NC, USA). The data are expressed as the mean \pm standard deviation of triplicate measurements. $\mathrm{P}<0.05$ was considered to indicate a statistically significant difference.

\section{Results}

GSK1838705A decreases glioma cell viability. Cellular proliferation is regulated by the cooperative action of various 
A

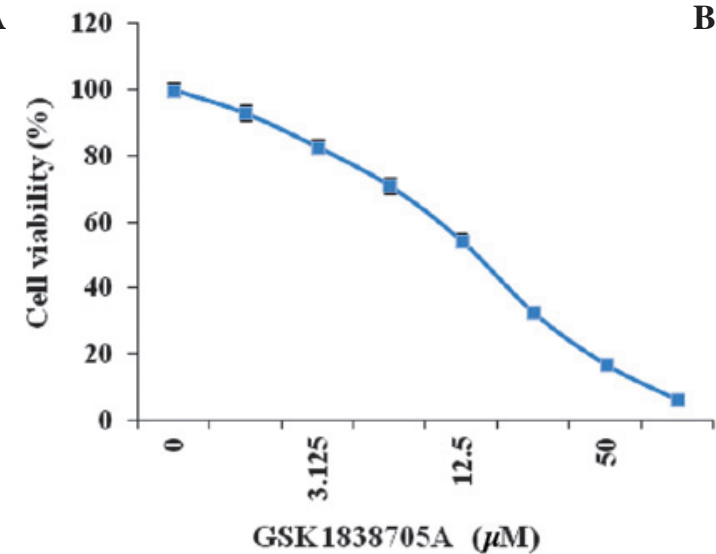

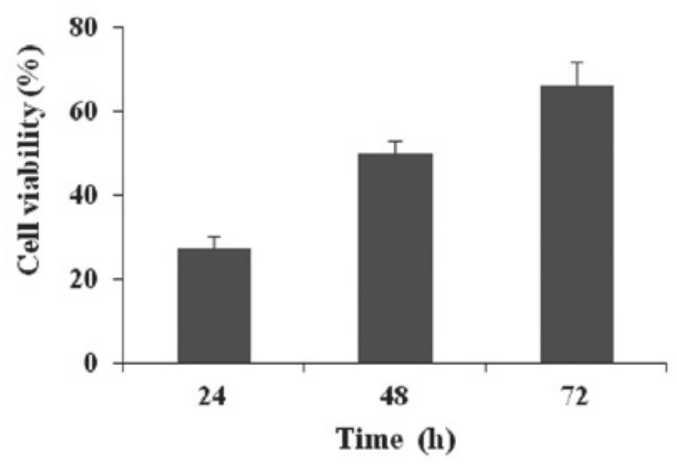

Figure 1. GSK1838705A reduces glioma cell viability. (A) U87MG cells were treated with DMSO or GSK1838705A (1.56-100 $\mu$ M) for 72 h, followed by measurement of cell viability. The viability of the cells treated with DMSO was set to $100 \%$. (B) U87MG cells were treated with $20 \mu \mathrm{M}$ GSK1838705A for 24,48 or $72 \mathrm{~h}$, followed by measurement of cell viability ( $\mathrm{P}<0.05,48$ and 72 vs. $24 \mathrm{~h}$ ). The data are expressed as the mean \pm standard deviation. DMSO, dimethyl sulfoxide.

growth stimulatory and inhibitory signals. IGF signaling favors cell survival by activating downstream signaling transduction cascades, including the Ras, MAPK, PI3K and AKT pathways $(8,20)$. To determine whether the suppression of IGF signaling affected cells proliferation in the present study, the U87MG glioma cells were treated with different concentrations of GSK1838705A for $72 \mathrm{~h}$ and the cell viability was measured. A dose-dependent inhibition of glioma cell viability was observed following treatment with GSK1838705A (Fig. 1A). To determine the onset of drug action, the U87MG cells were treated with GSK1838705A (20 $\mu \mathrm{M})$ for 24,48 or $72 \mathrm{~h}$, followed by the measurement of cell viability. The results indicated an early onset for the inhibitory effect of GSK1838705A, which was observed $24 \mathrm{~h}$ following treatment (Fig. 1B).

GSK1838705A induces the apoptosis of glioma cells. Tumor cells often evolve mechanisms to evade or antagonize programmed cell death, and IGF is a potent pro-survival factor, which increases cell proliferation, therefore activation of IGF signaling provides cells with a growth advantage (8). The present study investigated whether inhibiting IGF signaling via treatment of glioma cells with GSK1838705A induced apoptosis in the glioma cell. The sub-G1 DNA content in cells was measured as an indicator of late stage apoptosis (21). Consistent with the results obtained in the measurement of cell viability, GSK1838705A caused an increase in sub-G1 DNA content in a dose-dependent manner, which confirmed that the inhibition of IGF signaling promoted apoptosis in the affected cells (Fig. 2A). Apoptosis-induced condensation and fragmentation of DNA was visualized following nuclear staining. Compared with the untreated cells, the cells treated with GSK1838705A exhibited marked apoptosis, as shown in Fig. 2B. Similar to the results shown in Fig. 2A, the percentage of cells with condensed/fragmented DNA increased proportionally with increasing concentrations of GSK1838705A (Fig. 2C).

GSK1838705A inhibits the migration of glioma cells. Malignant tumor cells, including gliomas, are capable of migrating and invading to a secondary site through the processes of angiogenesis and metastasis, in which IGF signaling is important $(8,13)$. Therefore, IGF/IGF-IR is an attractive target in cancer therapeutics. The present study further investigated the effect of IGF inhibition on glioma cell migration. U87MG cells, in the presence or absence of GSK1838705A, were induced to migrate in a Transwell assay. As shown in Fig. 3A and B, an inhibitory effect of GSK1838705A on cellular migration was observed after $8 \mathrm{~h}$ of treatment at concentrations as low as $3.75 \mu \mathrm{M}$ and reached its peak effect at $15 \mu \mathrm{M}$. The reduced number of migrating cells was not a result of apoptosis due to GSK1838705A treatment itself, as the duration of treatment was significantly shorter and the concentrations of GSK1838705A were significantly lower compared with those used to examine the cell viability and apoptosis (Figs. 1 and 2).

GSK1838705A suppresses tumor cell growth in vivo. Subsequent to the results obtained from the in vitro investigations, the present study investigated the antitumor efficacy of GSK1838705A in vivo, in which U87MG cells were injected into athymic nude mice. Following successful inoculation, GSK1838705A (4 or $8 \mathrm{mg} / \mathrm{kg}$ ) was administered once daily and the tumor volumes were measured every other day. GSK1838705A significantly inhibited the growth of the tumor mass. Treatment with GSK1838705A at 4 or $8 \mathrm{mg} / \mathrm{kg}$ resulted in reductions of $\sim 45$ and $85 \%$ in tumor volume, respectively, 11 days after the first administration (Fig. 4A). The antitumor efficacy of GSK1838705A was consistent with the concentration used. No significant weight loss was observed in either treatment group during the course of treatment, indicating that the concentrations of GSK1838705A used were well tolerated by the recipient mice, and no significant cytotoxicity accompanied the GSK1838705A treatment (Fig. 4B). Consistent with the results of the in vitro investigations, GSK1838705A induced significant apoptosis in the tumor cells. Following treatment of tumors with $8 \mathrm{mg} / \mathrm{kg}$ GSK1838705A, significant DNA fragmentation was detected using a TUNEL assay and nuclear staining (Fig. 4C). Taken together, these results provided clear evidence indicating that GSK1838705A effectively suppressed the growth of the tumor in vivo by inducing apoptosis of the tumor cells. 
A

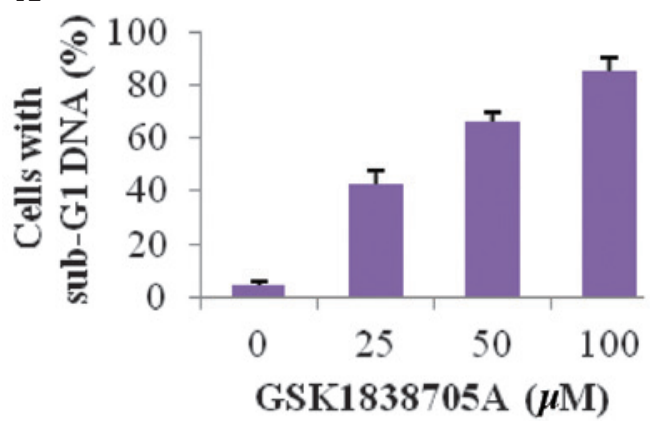

B

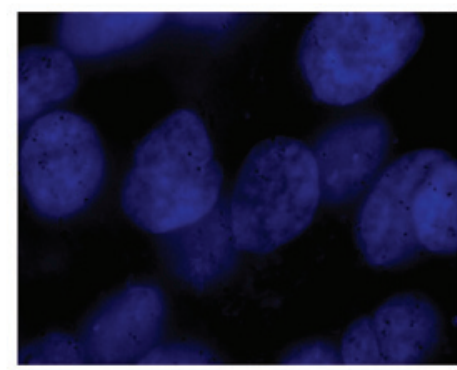

GSK1838705A $(100 \mu \mathrm{M})$

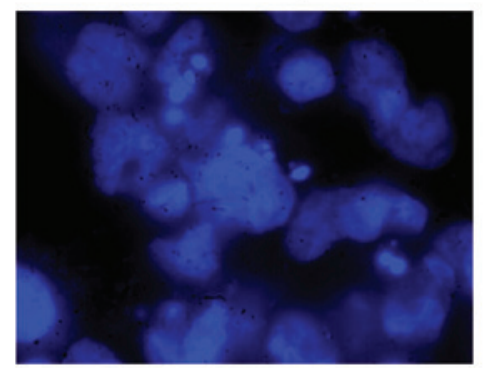

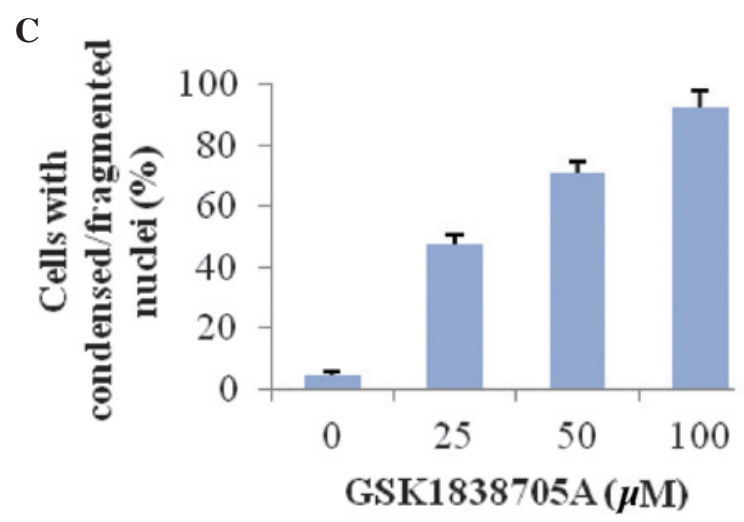

Figure 2. GSK1838705A induces the apoptosis of glioma cells. (A) U87MG cells were treated with DMSO or GSK1838705A at the indicated concentrations for $48 \mathrm{~h}$, followed by the analysis of sub-G1 DNA content. (B) U87MG cells were incubated with DMSO or $100 \mu \mathrm{M}$ GSK1838705A for $48 \mathrm{~h}$. The nuclei were stained with Hoechst and analyzed using fluorescent microscopy (magnification, x100). Representative images are shown. (C) Number of cells with condensed/fragmented nuclei, quantified by counting in seven randomly-selected fields, from which the average percentage was calculated. $\mathrm{P}<0.05,25,50$ and 100 vs. $0 \mu \mathrm{M}$. The data are expressed as the mean \pm standard deviation DMSO, dimethyl sulfoxide.

A
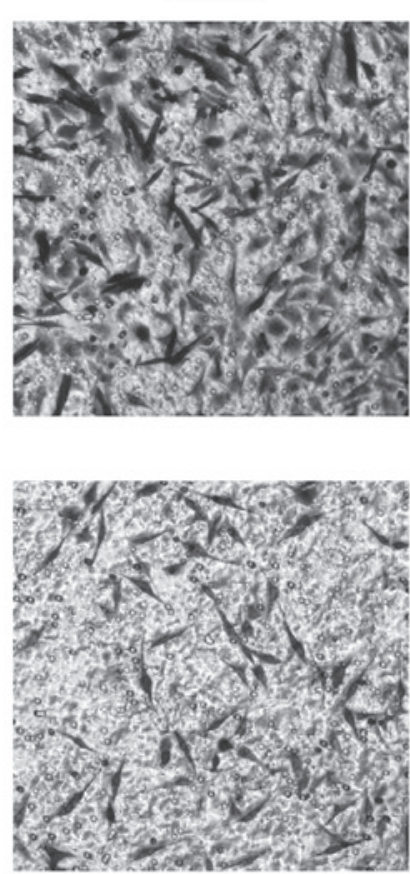

GSK1838705A $(7.5 \mu \mathrm{M})$
GSK1838705A $(3.75 \mu \mathrm{M})$
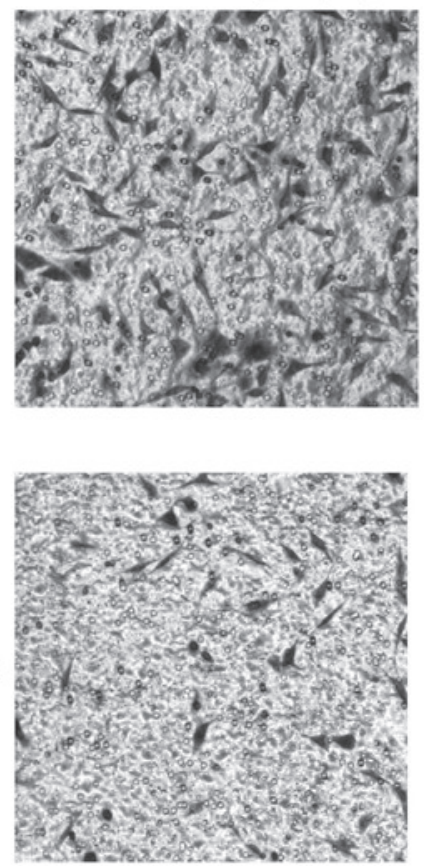

GSK1838705A $(15 \mu \mathrm{M})$
B

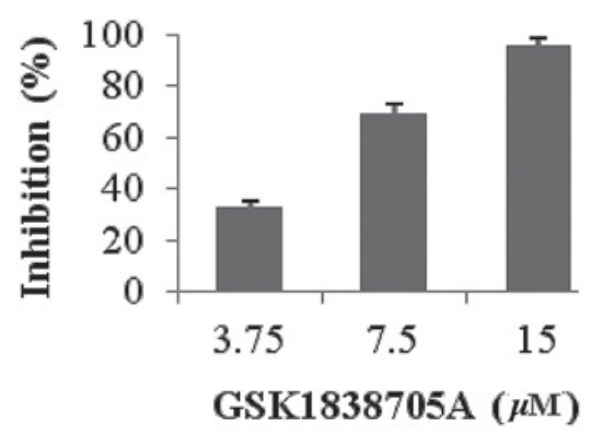

Figure 3. GSK1838705A inhibits the migration of glioma cells. (A) U87MG cells were treated with dimethyl sulfoxide or GSK1838705A (3.75, 7.5 or $15 \mu \mathrm{M}$ ) for $8 \mathrm{~h}$. Non-migrating cells on the upper surface of the filter were removed and migrated cells on the lower side were stained, following which images were captured (magnification, x40). Ten fields from each group were randomly selected, and migrated cells appeared to be dark and spindle-shaped after crystal violet staining. Representative images are shown. (B) Migrating cells were lysed and colorimetric determination was assessed at $595 \mathrm{~nm}$. Quantification of the inhibition is shown. The data are expressed as the mean \pm standard deviation. $\mathrm{P}<0.05,15$ and 7.5 vs. $3.75 \mu \mathrm{M}$. 
A

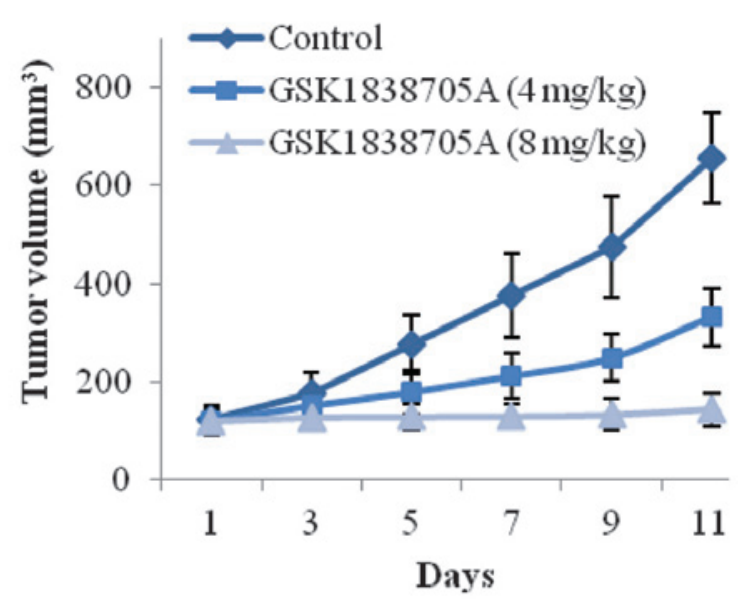

C
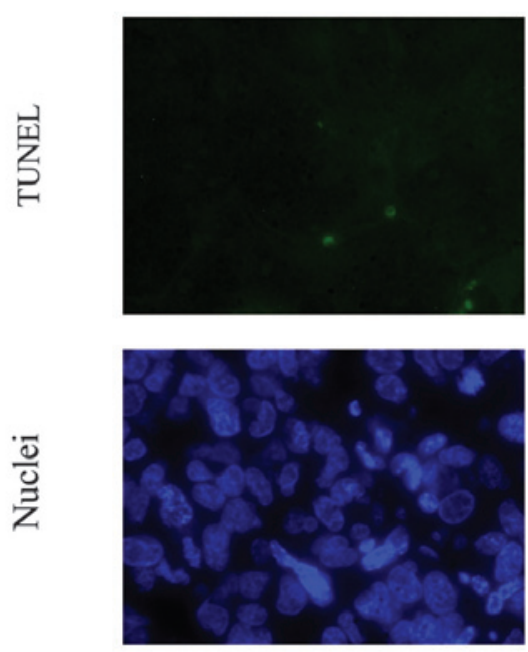

B

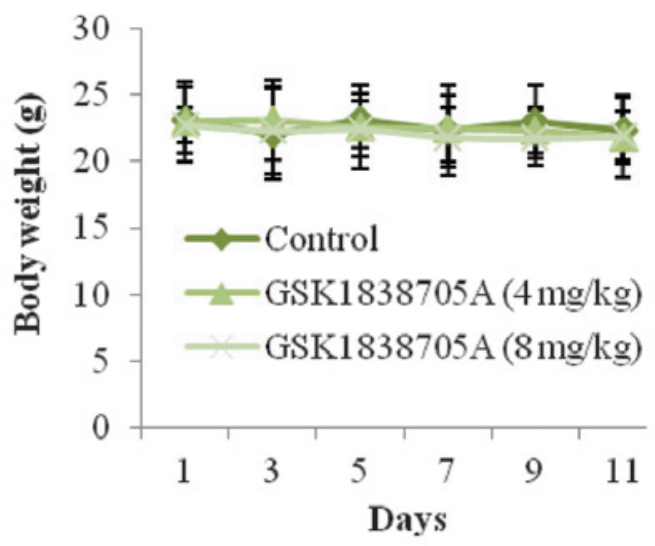

GSK1838705A ( 8 mg $/ \mathrm{kg})$
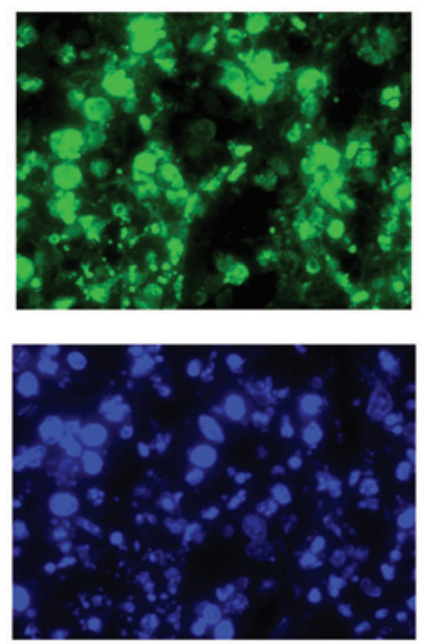

Figure 4. GSK1838705A suppresses glioma tumor growth and induces apoptosis in vivo. (A) Following inoculation of U87MG cells, formulated vehicle control or GSK1838705A ( 4 and $8 \mathrm{mg} / \mathrm{kg}$ ) was injected into the corresponding group of nude mice ( $\mathrm{n}=6 / \mathrm{group}$ ) once daily. The tumors were measured every other day for 11 days and the tumor volumes were calculated. Starting on day 7 , the differences between the treatment groups (4 and $8 \mathrm{mg} / \mathrm{kg}$ ) and the vehicle control group were significant $(\mathrm{P}<0.05)$. (B) Body weights of the mice during the course of treatment were measured as an indication of significant cytotoxic effects. The data are expressed as the mean \pm standard deviation. No significant differences are observed between any two of the groups during the course of treatment. (C) At the end of treatment, the tumors were harvested. GSK1838705A (8 mg/kg) induced the apoptosis of tumor cells in vivo, determined using a TUNEL assay (green) and nuclear staining with Hoechst (blue). Representative images are shown (magnification, x40). TUNEL, terminal deoxynucleotidyl transferase dUTP nick end labeling.

\section{Discussion}

Dysregulated signaling pathways have been implicated in the tumorigenesis and angiogenesis of a wide variety of human malignancies. The critical elements involved in signal transduction, including surface receptors, kinases, adaptor proteins and various signaling molecules, have been identified with advances in biological research. The IGF signaling pathway represents an example of the transformation of a functional growth regulator into a tumor promoter when disturbance occurs (8). Since IGF has a common mode of action in a wide range of tumor types, IGF signaling is an attractive therapeutic target for cancer therapy. In human glioma, overexpression of IGF-1, IGF-2 and IGF-IR has been identified, and the paracrine stimulatory loop promotes tumor growth and invasion (22). By selecting biological inhibitors with a specific targeting spectrum, effective therapeutic agents can be directed to a targeted population with higher sensitivity. The high-grade gliomas, glioblastoma in particular, are aggressive and the clinical outcomes are particularly poor. Molecular examinations of glioblastoma cells have revealed frequent loss of the phosphatase and tensin homologue (PTEN), a tumor suppressor gene, which results in increased activation of the PI3K/AKT signaling pathway $(23,24)$. Using the PTEN-deficient human U87MG glioma cell line in the present study enabled manipulation of the activity of AKT through alteration of the upstream IGF signaling (25). The present study demonstrated the use of GSK1838705A, a novel kinase inhibitor of IGF-IR, as a therapeutic possibility for the treatment of human glioma.

Previous studies in different tumor cell lines have revealed the importance of GSK1838705A in the inhibition of cellular proliferation $(18,19)$. The results from the present study provided additional evidence to demonstrate the efficacy of GSK1838705A in inhibiting the growth of glioma tumor cells. Treatment with GSK1838705A decreased the survival and induced the apoptosis of U87MG glioma cells in a dose-dependent manner. Previous investigation has also demonstrated the role of IGF signaling in the assembly of 
vascular networks, which are required for angiogenesis and metastasis. IGF-1 was reported to stimulate the expression of vascular endothelial growth factor (VEGF) through the AKT pathway in a thyroid carcinoma model (26). The perivascular glioma cells exhibit higher expression levels of IGF-IR than the cells in other tumor zones $(13,17)$. In the present study, GSK1838705A-treated U87MG cells exhibited reduced migratory activity in response to chemoattractants, therefore, a beneficial effect of GSK1838705A on the suppression of angiogenesis and tumor invasion is expected. Furthermore, administration of GSK1838705A significantly inhibited the growth of U87MG tumor cells in vivo. The present study demonstrated for the first time, to the best of our knowledge, the antitumor activity of GSK1838705A in glioma cells.

Tumorigenesis involves multiple steps and receives contribution from different gene products and signaling pathways. Tumor cells usually exhibit a high degree of molecular heterogeneity, which leads to different sensitivities to targeted chemotherapies and the emergence of chemoresistance. Future treatment approaches to combat life-threatening malignancies, including glioma, are most likely to be combinational strategies, in which multi-target based chemotherapies against various disease-associated pathways increase the effects of conventional cytotoxic drugs and radiation therapy. Therefore, in glioma therapy, future investigations are required to identify the effective combination of targeted therapies. The inhibition of IGF signaling by GSK1838705A may be combined with suppression of other growth factor pathways, including the epidermal growth factor (EGF) or VEGF pathways, to assess the antitumor efficacy. It is hypothesized that innovative targeted therapies are likely to offer significant clinical benefit and lead to the development of personalized medicine.

\section{References}

1. Ohgaki $\mathrm{H}$ and Kleihues P: Population-based studies on incidence, survival rates and genetic alterations in astrocytic and oligodendroglial gliomas. J Neuropathol Exp Neurol 64: 479-489, 2005.

2. Smoll NR, Gautschi OP, Schatlo B, Schaller K and Weber DC: Relative survival of patients with supratentorial low-grade gliomas. Neuro Oncol 14: 1062-1069, 2012.

3. SpinelliGP,Miele E,LoRusso G,Miscusi M,Codacci-Pisanelli G, Petrozza V, Papa A, Frati L, Della Rocca C, Gulino A and Tomao S: Chemotherapy and target therapy in the management of adult high-grade gliomas. Curr Cancer Drug Targets 12: 1016-1031, 2012.

4. Wang $\mathrm{Y}$ and Jiang $\mathrm{T}$ : Understanding high grade glioma: Molecular mechanism, therapy and comprehensive management. Cancer Lett 331: 139-146, 2013.

5. Newlands ES, Stevens MF, Wedge SR, Wheelhouse RT and Brock C: Temozolomide: A review of its discovery, chemical properties, pre-clinical development and clinical trials. Cancer Treat Rev 23: 35-61, 1997.

6. Stupp R, Hegi ME, Mason WP, van den Bent MJ, Taphoorn MJ, Janzer RC, Ludwin SK, Allgeier A, Fisher B, Belanger $\mathrm{K}$, et al: Effects of radiotherapy with concomitant and adjuvant temozolomide versus radiotherapy alone on survival in glioblastoma in a randomised phase III study: 5-year analysis of the EORTC-NCIC trial. Lancet Oncol 10: 459-466, 2009.

7. Cao Z, Liu LZ, Dixon DA, Zheng JZ, Chandran B and Jiang BH: Insulin-like growth factor-I induces cyclooxygenase-2 expression via PI3K, MAPK and PKC signaling pathways in human ovarian cancer cells. Cell Signal 19: 1542-1553, 2007.

8. Weroha SJ and Haluska P: The insulin-like growth factor system in cancer. Endocrinol Metab Clin North Am 41: 335-350, vi, 2012.
9. Jenkins PJ, Frajese V, Jones AM, Camacho-Hubner C, Lowe DG, Fairclough PD, Chew SL, Grossman AB, Monson JP, Besser GM, et al: Insulin-like growth factor I and the development of colorectal neoplasia in acromegaly. J Clin Endocrinol Metab 85: 3218-3221, 2000.

10. Lukanova A, Lundin E, Toniolo P, Micheli A, Akhmedkhanov A, Rinaldi S, Muti P, Lenner P, Biessy C, Krogh V, et al: Circulating levels of insulin-like growth factor-I and risk of ovarian cancer. Int J Cancer 101: 549-554, 2002.

11. Roddam AW, Allen NE, Appleby P, Key TJ, Ferrucci L, Carter HB, Metter EJ, Chen C, Weiss NS, Fitzpatrick A, et al: Insulin-like growth factors, their binding proteins and prostate cancer risk: Analysis of individual patient data from 12 prospective studies. Ann Intern Med 149: 461-471, W83-W88, 2008.

12. Endogenous Hormones and Breast Cancer Collaborative Group, Key TJ, Appleby PN, Reeves GK and Roddam AW: Insulin-like growth factor 1 (IGF1), IGF binding protein 3 (IGFBP3) and breast cancer risk: Pooled individual data analysis of 17 prospective studies. Lancet Oncol 11: 530-542, 2010.

13. Hirano H, Lopes MB, Laws ER Jr, Asakura T, Goto M, Carpenter JE, Karns LR and VandenBerg SR: Insulin-like growth factor-1 content and pattern of expression correlates with histopathologic grade in diffusely infiltrating astrocytomas. Neuro Oncol 1: 109-119, 1999.

14. Resnicoff M, Sell C, Rubini M, Coppola D, Ambrose D, Baserga $\mathrm{R}$ and Rubin R: Rat glioblastoma cells expressing an antisense RNA to the insulin-like growth factor-1 (IGF-1) receptor are nontumorigenic and induce regression of wild-type tumors. Cancer Res 54: 2218-2222, 1994.

15. Yin S, Girnita A, Strömberg T, Khan Z, Andersson S, Zheng H, Ericsson C, Axelson M, Nistér M, Larsson O, et al: Targeting the insulin-like growth factor-1 receptor by picropodophyllin as a treatment option for glioblastoma. Neuro Oncol 12: 19-27, 2010.

16. Gariboldi MB, Ravizza R and Monti E: The IGFR1 inhibitor NVP-AEW541 disrupts a pro-survival and pro-angiogenic IGF-STAT3-HIF1 pathway in human glioblastoma cells. Biochem Pharmacol 80: 455-462, 2010.

17. Schlenska-Lange A, Knüpfer H, Lange TJ, Kiess W and Knüpfer M: Cell proliferation and migration in glioblastoma multiforme cell lines are influenced by insulin-like growth factor I in vitro. Anticancer Res 28: 1055-1060, 2008.

18. Sabbatini P, Korenchuk S, Rowand JL, Groy A, Liu Q, Leperi D, Atkins C, Dumble M, Yang J, Anderson K, et al: GSK1838705A inhibits the insulin-like growth factor-1 receptor and anaplastic lymphoma kinase and shows antitumor activity in experimental models of human cancers. Mol Cancer Ther 8: 2811-2820, 2009.

19. Bao NR, Lu M, Bin FW, Chang ZY, Meng J, Zhou LW, Guo T and Zhao JN: Systematic screen with kinases inhibitors reveals kinases play distinct roles in growth of osteoprogenitor cells. Int J Clin Exp Pathol 6: 2082-2091, 2013.

20. Anisimov VN and Bartke A: The key role of growth hormone-insulin-IGF-1 signaling in aging and cancer. Crit Rev Oncol Hematol 87: 201-223, 2013.

21. Nicoletti I, Migliorati G, Pagliacci MC, Grignani F and Riccardi C: A rapid and simple method for measuring thymocyte apoptosis by propidium iodide staining and flow cytometry. J Immunol Methods 139: 271-279, 1991.

22. Trojan J, Cloix JF, Ardourel MY, Chatel M and Anthony DD: Insulin-like growth factor type I biology and targeting in malignant gliomas. Neuroscience 145: 795-811, 2007.

23. Freeman DJ, Li AG, Wei G, Li HH, Kertesz N, Lesche R, Whale AD, Martinez-Diaz H, Rozengurt N, Cardiff RD, et al: PTEN tumor suppressor regulates p53 protein levels and activity through phosphatase-dependent and -independent mechanisms. Cancer Cell 3: 117-130, 2003.

24. Choe G, Horvath S, Cloughesy TF, Crosby K, Seligson D, Palotie A, Inge L, Smith BL, Sawyers CL and Mischel PS: Analysis of the phosphatidylinositol 3'-kinase signaling pathway in glioblastoma patients in vivo. Cancer Res 63: 2742-2746, 2003.

25. Ishii N, Maier D, Merlo A, Tada M, Sawamura Y, Diserens AC and Van Meir EG: Frequent co-alterations of TP53, p16/CDKN2A, p14ARF, PTEN tumor suppressor genes in human glioma cell lines. Brain Pathol 9: 469-479, 1999.

26. Poulaki V, Mitsiades CS, McMullan C, Sykoutri D, Fanourakis G, Kotoula V, Tseleni-Balafouta S, Koutras DA and Mitsiades N: Regulation of vascular endothelial growth factor expression by insulin-like growth factor I in thyroid carcinomas. J Clin Endocrinol Metab 88: 5392-5398, 2003. 